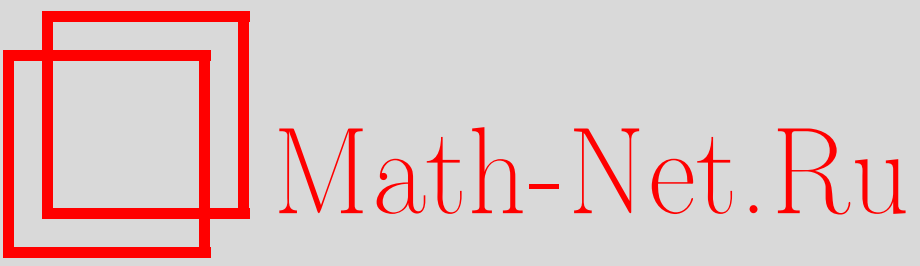

С. Г. Клементьев, В. М. Петроградский, О росте почти разрешимых алгебр Ли, УМH, 2005, том 60, выпуск 5, 165-166

DOI: https://doi.org/10.4213/rm1646

Использование Общероссийского математического портала Math-Net.Ru подразумевает, что вы прочитали и согласны с пользовательским соглашением

http://www.mathnet.ru/rus/agreement

Параметры загрузки:

IP: 44.207 .124 .84

26 апреля 2023 г., 14:38:37 


\title{
О РОСТЕ ПОЧТИ РАЗРЕШИМЫХ АЛГЕБР ЛИ
}

\author{
С. Г. КЛЕМЕНТЬЕВ, В. М. ПЕТРОГРАДСКИЙ
}

Обозначим $\ln ^{(1)} n=\ln n$ и $\ln ^{(q+1)} n=\ln \left(\ln ^{(q)} n\right), q=1,2, \ldots$. Аналогично определяем $\exp ^{(k)} x$. Рассмотрим последовательность функций $\Phi_{\alpha}^{q}(n), q=1,2, \ldots$, с параметром $\alpha \in \mathbb{R}^{+}$:

$$
\begin{array}{ll}
\Phi_{\alpha}^{1}(n)=\alpha, & \Phi_{\alpha}^{2}(n)=n^{\alpha}, \\
\Phi_{\alpha}^{3}(n)=\exp \left(n^{\alpha /(\alpha+1)}\right), & \Phi_{\alpha}^{q}(n)=\exp \left(\frac{n}{\left(\ln ^{(q-3)} n\right)^{1 / \alpha}}\right), \quad q=4,5, \ldots .
\end{array}
$$

Рассмотрим алгебру $A$ над полем $K$, порожденную конечным множеством $X$. Обозначим через $\gamma_{A}(X, n)$ размерность пространства, образованного всеми мономами из элементов $X$ длины не более $n$. Определим (верхнюю) размерность уровня $q, q=1,2, \ldots$ :

$\operatorname{Dim}^{q} A=\inf \left\{\alpha \in \mathbb{R}^{+} \mid \exists N: \gamma_{A}(X, n) \leqslant \Phi_{\alpha}^{q}(n), n \geqslant N\right\}$.

Нижняя размерность $\underline{\operatorname{Dim}}^{q} A$ определяется аналогично. Эти размерности обобщают размерность Гельфанда-Кириллова [1]. Данные размерности были введены для изучения субэкспоненциального роста конечно порожденных разрешимых групп и алгебр Ли [2], [3].

Для алгебры Ли $L$ рассмотрим цепочку коммутантов: $L^{(0)}=L$ и $L^{(i+1)}=\left[L^{(i)}, L^{(i)}\right]$, $i \geqslant 0$. Алгебра Ли назьвается разрешимой ступени $q$, если $L^{(q)}=0$. Рассмотрим класс всех алгебр Ли, разрешимых ступени $q$ и порожденных $k$ элементами. В нем существует универсальньй объект $F\left(\mathbf{A}^{q}, k\right)$, называемьй свободной разрешимой алгеброй Ли ступени $q$ и ранга $k$ [4].

Teоpema 1 [2], [3]. Пусть $L=F\left(\mathbf{A}^{q}, k\right)$, где $k \geqslant 2, q \geqslant 1$. Тогда $\underline{\operatorname{Dim}}^{q} L=\operatorname{Dim}^{q} L=k$.

Целью настоящей заметки является следующая субэкспоненциальная верхняя оценка на рост почти разрешимых алгебр Ли.

ТеОРема 2. Пусть в конечно порожденной алгебре Ли L существует подалгебра $H \subseteq L$ конечной коразмерности, которая разрешима ступени $q$. Тогда $\operatorname{Dim}^{q+1} L \leqslant$ $\operatorname{dim}_{K}(L / H)$.

Из теоремы 1 следует точность этой оценки. Далее $f(z)=\sum_{n=0}^{\infty} a_{n} z^{n}$ - функция, аналитичная в единичном круге. Обозначим $\mathrm{M}_{f}(r)=\max _{|z|=r}|f(z)|$ для $0 \leqslant r<1$.

ЛЕмма 1 [5]. Зафиксируем $\alpha \in \mathbb{R}^{+}, \sigma \in[0 ; \infty] ;$ обозначим $\kappa(\sigma)=(1+1 / \alpha)(\sigma \alpha)^{1 /(1+\alpha)}$. Тогда $\overline{\lim }_{r \rightarrow 1-0}(1-r)^{\alpha} \ln \mathrm{M}_{f}(r)=\sigma \Longleftrightarrow \varlimsup_{n \rightarrow \infty} n^{-\alpha /(\alpha+1)} \ln \left|a_{n}\right|=\kappa(\sigma)$.

Лемма 2 [5]. Зафиксируем $\alpha \in \mathbb{R}^{+}, s \in \mathbb{N}$. Тогда $\varlimsup_{r \rightarrow 1-0}(1-r)^{\alpha} \ln ^{(s+1)} \mathrm{M}_{f}(r)=\left(\varlimsup_{\lim } \rightarrow \infty\left(\ln \left|a_{n}\right| \cdot\left(\ln ^{(s)} n\right)^{1 / \alpha} / n\right)\right)^{\alpha}$.

Нас интересуют ряды $\phi(t)=\sum_{n=1}^{\infty} a_{n} t^{n}$ с целшми неотрицательными коэффиициентами, аналитичные в единичном круге; в этом случае $\mathrm{M}_{\phi}(r)=\phi(r)$. Леммы 3 и 4 описывают действие на таких рядах следующего оператора $\mathscr{E}: \phi(t)=\sum_{n=1}^{\infty} a_{n} t^{n} \longmapsto \prod_{n=1}^{\infty}\left(1-t^{n}\right)^{-a_{n}}$.

Лемма 3 [5]. Пусть $\lim _{t \rightarrow 1-0}(1-t)^{\alpha} \phi(t)=A$, где $A>0 u \alpha \geqslant 1$. Тогда $\lim _{t \rightarrow 1-0}(1-t)^{\alpha} \ln \mathscr{E}(\phi(t))=A \zeta(\alpha+1)$, где $\zeta(s)-$ дзета-функиия Римана.

Лемма 4 [5]. Пусть $\lim _{t \rightarrow 1-0}(1-t)^{\alpha} \ln ^{(q)} \phi(t)=A$, где $A>0 u q \in \mathbb{N}$. Тогда $\lim _{t \rightarrow 1-0}(1-t)^{\alpha} \ln ^{(q+1)} \mathscr{E}(\phi(t))=A$.

Назовем множество $X=\bigcup_{m=1}^{\infty} X_{m}$, где $\left|X_{m}\right|<\infty$, градуированным. Расширим наши определения на случай градуированного множества, где элементы $X_{m}$ имеют вес $m$ при вычислении функций роста. Определим $\lambda_{A}(X, n)=\gamma_{A}(X, n)-\gamma_{A}(X, n-1)$ для $n \geqslant 1$. Определим также ряд Гильберта $\mathscr{H}_{X}(A, t)=\sum_{n=1}^{\infty} \lambda_{A}(X, n) t^{n}$. Положим $\mathscr{H}(X, t)=\sum_{n=1}^{\infty}\left|X_{n}\right| t^{n}$. Следующий результат - это частньй случай следствия 4.1 в [6].

Работа вьполнена при частичной поддержке РФФИ (грант № 04-01-00739), второй автор частично поддержан фондом Гумбольдта. 
Теорема 3 [6]. Пусть $L=F\left(\mathbf{A}^{q}, Y\right)$ - свободная разрешимая алгебра Ли ступени $q$, порожденная градуированным множеством $Y$. Обозначим $g_{1}(t)=\mathscr{H}(Y, t)$ и $g_{i}(t)=$ $g_{i-1}(t)+1+(\mathscr{H}(Y, t)-1) \mathscr{E}\left(g_{i-1}(t)\right)$ для $i=2, \ldots, q$. Тогда $\mathscr{H}_{Y}(L, t)=g_{q}(t)$.

ДоКАЗАТЕльство теОРемы 2. Пусть $L$ порождена множеством $Z=\left\{z_{1}, \ldots, z_{k}\right\}$. По условию $\operatorname{dim}_{K} L / H=N<\infty$ и $H^{(q)}=0$. Пусть $F=F(X)$ - свободная алгебра Ли, порожденная $X=\left\{x_{1}, \ldots, x_{k}\right\}$. Имеем эпиморфизм $\phi: F \rightarrow L, \phi\left(x_{i}\right)=z_{i}$ для $i=1, \ldots, k$. Обозначим $D=\phi^{-1}(H)$ и $G=\operatorname{Ker} \phi$. Тогда $\operatorname{dim}_{K} F / D=N, F / G \cong L$ и $D^{(q)} \subseteq G$.

Рассмотрим фильтрацию $F^{1} \subseteq F^{2} \subseteq \cdots$, заданную степенью относительно $X$. Для подпространства $V \subseteq F$ пусть gr $V=\bigoplus_{n=1}^{\infty} \operatorname{gr}_{n} V \subseteq F$ обозначает ассоциированное градуированное пространство. Подалгебра gr $D \subseteq F$ является свободной по теореме Ширшова-Витта [4]. Пусть $\bar{Y}=\bigcup_{n=1}^{\infty} \bar{Y}_{n}$ - однородное свободное порождающее множество для $\operatorname{gr} D$, где $\bar{Y}_{n} \subset \operatorname{gr}_{n} F$. Пусть $\mathscr{H}_{X}(\bar{Y}, t)=\sum_{n=1}^{\infty}\left|\bar{Y}_{n}\right| t^{n}$, тогда имеет место аналог формулы Шрайера [7], [6]:

$$
\mathscr{H}_{X}(\bar{Y}, t)-1=(k t-1) \mathscr{E}\left(\mathscr{H}_{X}(F / \text { gr } D, t)\right),
$$

где $\mathscr{H}_{X}(F / \operatorname{gr} D, t)=\sum_{n=1}^{\infty} c_{n} t^{n}$ - многочлен, так как $\sum_{n} c_{n}=\operatorname{dim} F / \operatorname{gr} D=\operatorname{dim} F / D=N$.

Рассмотрим цепочку $F \supseteq D \supseteq G \supseteq D^{(q)}$. Для роста алгебры $L$ получаем оценку $\gamma_{L}(Z, n)=\gamma_{F / G}(X, n)=\sum_{j=1}^{\bar{n}} \lambda_{F / G}(X, j)=\sum_{j=1}^{n} \operatorname{dim}_{j}(F / G) \leqslant \sum_{j=1}^{n} \operatorname{dim} \operatorname{dr}_{j}(F / D)+$ $\sum_{j=1}^{n} \operatorname{dimgr}_{j}\left(D / D^{(q)}\right) \leqslant N+\gamma_{\operatorname{gr}\left(D / D^{(q)}\right)}(\bar{Y}, n), n \in \mathbb{N}$. Заметим, что gr $D / D^{(q)} \cong F\left(\mathbf{A}^{q}, \bar{Y}\right)$. Обозначим $\mathscr{H}\left(F\left(\mathbf{A}^{q}, \bar{Y}\right), t\right)=\sum_{n=1}^{\infty} d_{n} t^{n}$, пусть $\bar{d}_{n}=d_{1}+\cdots+d_{n}$ для $n \in \mathbb{N}$. Таким образом, имеем оценку $\gamma_{L}(n) \leqslant N+\bar{d}_{n}, n \in \mathbb{N}$, поэтому достаточно получить искомые оценки для $\bar{d}_{n}$.

Применим теорему 3 и (1) к алгебре $F\left(\mathbf{A}^{q}, \bar{Y}\right)$ :

$$
g_{1}(t)=\mathscr{H}_{X}(\bar{Y}, t)=1+(k t-1) \prod_{n \geqslant 1} \frac{1}{\left(1-t^{n}\right)^{c_{n}}}=\frac{A+o(1)}{(1-t)^{N}}, \quad t \rightarrow 1-0 .
$$

Имеем покоэффициентную и обычную оценки

$$
g_{i}(t) \leqslant g_{i-1}(t)+g_{1}(t) \cdot \mathscr{E}\left(g_{i-1}(t)\right), \quad 0 \leqslant t<1 ; \quad i=2, \ldots, q .
$$

Для $i=2$ применяем лемму 3 к (3) и $(2)$; для $i=3, \ldots, q$ несколько раз применяем лемму 4 :

$$
g_{p}(t)=\exp ^{(p-1)}\left(\frac{A \zeta(N+1)+o(1)}{(1-t)^{N}}\right), t \rightarrow 1-0 ; \quad p=2, \ldots, q .
$$

По теореме $3 \mathscr{H}\left(F\left(\mathbf{A}^{q}, \bar{Y}\right), t\right)=g_{q}(t)$. Получим окончательные оценки в зависимости от $q$. Случай $q=1$ : из $(2)$ вытекает, что $\bar{d}_{n} \approx C n^{N}, n \rightarrow \infty$, для некоторой константы $C$; следовательно, $\operatorname{Dim}^{2} L \leqslant N$. Рассмотрим $q=2$. Из (4) по лемме 1 получаем

$$
\begin{gathered}
\varlimsup_{n \rightarrow \infty} n^{-N /(N+1)} \ln d_{n}=\kappa(\zeta(N+1) A)=(1+1 / N)(\zeta(N+1) A N)^{1 /(N+1)}=C_{0} \\
d_{n} \leqslant \exp \left(\left(C_{0}+o(1)\right) n^{N /(N+1)}\right), \quad n \rightarrow \infty .
\end{gathered}
$$

Такая же оценка имеет место для $\bar{d}_{n}$. Получаем $\operatorname{Dim}^{3} L \leqslant N$. Аналогично, в случае $q \geqslant 3$ мы применяем лемму 2 к (4) и получаем $\operatorname{Dim}^{q+1} L \leqslant N$.

\section{СПИСОК ЛИТЕРАТУРЫ}

[1] I. M. Gelfand, A. A. Kirillov // Inst. Hautes Études Sci. Publ. Math. 1966. V. 31. P. 506-523. [2] В. М. Петроградский // УМН. 1993. Т. 48. № 5. С. 181-182. [3] V. M. Petrogradsky // J. Algebra. 1996. V. 179. № 2. Р. 459-482. [4] Ю. А. Бахтурин. Тождества в алгебрах Ли. М.: Наука, 1985. [5] V. M. Petrogradsky // Internat. J. Algebra Comput. 1999. V. 9. № 2. P. 179-212. [6] V. M. Petrogradsky // Discrete Math. 2002. V. 246. № 1-3. P. 269-284. [7] V. M. Petrogradsky // Arch. Math. 2000. V. 75. P. 16-28. 